\title{
Phase transitions inK2ZnCl4
}

\section{Citation}

Lu, H. M., and J. R. Hardy. 1992. "Phase Transitions inK2ZnCl4." Physical Review B 46 (13) (October 1): 8582-8585. doi:10.1103/physrevb.46.8582.

\section{Published Version}

doi:10.1103/PhysRevB.46.8582

\section{Permanent link}

http://nrs.harvard.edu/urn-3:HUL.InstRepos:32416127

\section{Terms of Use}

This article was downloaded from Harvard University's DASH repository, and is made available under the terms and conditions applicable to Other Posted Material, as set forth at http:// nrs.harvard.edu/urn-3:HUL.InstRepos:dash.current.terms-of-use\#LAA

\section{Share Your Story}

The Harvard community has made this article openly available.

Please share how this access benefits you. Submit a story.

Accessibility 
Phys. Rev. B 46, 8582 - 8585 (1992)

\title{
Phase transitions in $\mathrm{K}_{2} \mathrm{ZnCl}_{4}$
}

\author{
H. M. Lu and J. R. Hardy
}

Department of Physics and Center for Electro-Optics, University of Nebraska-Lincoln, Lincoln, Nebraska 68588-0111

\begin{abstract}
It is shown that the potential-energy surface in $\mathrm{K}_{2} \mathrm{ZnCl}_{4}$ contains a double-well structure, very similar to some of the other $A_{2} B X_{4}$ compounds (e.g., $\mathrm{K}_{2} \mathrm{SeO}_{4}, \mathrm{Rb}_{2} \mathrm{ZnCl}_{4}$ ), except that the double well is much deeper and broader, giving rise to a highly disordered high-temperature phase as observed experimentally. A lattice-dynamics study of the Pna2 $2_{1}$ structure shows an instability with the wave vector $\mathrm{q}=0.5 \mathrm{~b}^{*}+(0.5 \pm \delta) \mathrm{c}^{*}$, providing an explanation to the incommensurate phase transition reported recently.
\end{abstract}

(C)1992 The American Physical Society

URL: http://link.aps.org/doi/10.1103/PhysRevB.46.8582

DOI: $10.1103 /$ PhysRevB.46.8582 


\title{
Brief Reports
}

Brief Reports are accounts of completed research which, while meeting the usual Physical Review standards of scientific quality, do not warrant regular articles. A Brief Report may be no longer than four printed pages and must be accompanied by an abstract. The same publication schedule as for regular articles is followed, and page proofs are sent to authors.

\section{Phase transitions in $\mathrm{K}_{2} \mathrm{ZnCl}_{4}$}

\author{
H. M. Lu and J. R. Hardy \\ Department of Physics and Center for Electro-Optics, University of Nebraska-Lincoln, \\ Lincoln, Nebraska 68588-0111
}

(Received 16 March 1992)

\begin{abstract}
It is shown that the potential-energy surface in $\mathrm{K}_{2} \mathrm{ZnCl}_{4}$ contains a double-well structure, very similar to some of the other $A_{2} B X_{4}$ compounds (e.g., $\mathrm{K}_{2} \mathrm{SeO}_{4}, \mathrm{Rb}_{2} \mathrm{ZnCl}_{4}$ ), except that the double well is much deeper and broader, giving rise to a highly disordered high-temperature phase as observed experimentally. A lattice-dynamics study of the $P$ na $2_{1}$ structure shows an instability with the wave vector $\mathbf{q}=0.5 \mathbf{b}^{*}+(0.5 \pm \delta) \mathbf{c}^{*}$, providing an explanation to the incommensurate phase transition reported recently.
\end{abstract}

For a long time, $\mathrm{K}_{2} \mathrm{ZnCl}_{4}$ has been considered to have the same phase sequence as $\mathrm{Rb}_{2} \mathrm{ZnCl}_{4}{ }^{1}$ It has the $\beta$ $\mathrm{K}_{2} \mathrm{SO}_{4}$ structure (Pnam) at high temperatures, like many other members in the family of the $A_{2} B X_{4}$ compounds. At $553 \mathrm{~K}\left(T_{i}\right)$, it transforms to an incommensurate phase with the incommensurate modulation vector $\mathbf{q}=\left[(1-\delta) \mathbf{a}^{*}\right] / 3$, where $\mathbf{a}^{*}$ is the first reciprocal-lattice vector along the [100] direction and $\delta \ll 1$. At $403 \mathrm{~K}$ $\left(T_{c 1}\right)$ the incommensurate modulation disappears and the system locks into a ferroelectric superstructure (Pna $\left.2_{1}\right)$ whose a axis is triple that of the Pnam phase. When cooled further, it undergoes another transition at $145 \mathrm{~K}$ $\left(T_{c 2}\right)$ to a monoclinic structure ( $\left.C 1 c 1\right)$. However, it was found recently that $\mathrm{K}_{2} \mathrm{ZnCl}_{4}$ has another incommensurate phase just above the lower-temperature monoclinic phase, while $\mathrm{Rb}_{2} \mathrm{ZnCl}_{4}$ has not. ${ }^{2,3}$ Reference 3 gave the temperature range for this phase as $140-144 \mathrm{~K}$ and revealed, by inelastic neutron scattering, a soft optic phonon with the wave vector $q=\left(0.5 b^{*}+(0.5 \pm \delta) c^{*}\right.$ responsible for the incommensurate phase transition.

In the present paper, we perform structural relaxation, molecular-dynamics simulation, and lattice-dynamics calculations of $\mathrm{K}_{2} \mathrm{ZnCl}_{4}$ using a recent first-principles approach to ionic molecular crystals. ${ }^{4,5}$ The method starts from $a b$ initio quantum chemistry calculations regarding the whole molecular ions, i.e., the $\mathrm{ZnCl}_{4}{ }^{2-}$ ions in the present case, as single entities and then uses the resultant realistic electron charge density to calculate the GordonKim pair potentials, ${ }^{6}$ thereby correctly treating the effects of intramolecular covalency on the intermolecular interactions. For the intramolecular interactions, which are beyond the Gordon-Kim model and the pairwise interaction scheme, a harmonic expansion is employed, with the expansion coefficients also determined by $a b$ initio quantum chemistry calculations. In our previous study of $\mathrm{Rb}_{2} \mathrm{ZnCl}_{4},{ }^{5}$ we have performed the quantum chemistry calculations for the free molecular ion $\mathrm{ZnCl}_{4}{ }^{2-}$ and therefore we can simply take those results, since the $\mathrm{ZnCl}_{4}{ }^{2-}$ ions take essentially the same form of modestly distorted tetrahedra in both $\mathrm{Rb}_{2} \mathrm{ZnCl}_{4}$ and $\mathrm{K}_{2} \mathrm{ZnCl}_{4}{ }^{7,8}$ Thus, we will use the same intramolecular harmonic expansions and we will use the same electron charge densities for the $\mathrm{Zn}$ and $\mathrm{Cl}$ atoms to compute the Gordon-Kim pair potentials, ${ }^{6}$ together with the charge density for the $\mathrm{K}^{+}$ion. ${ }^{9}$ Considering the good and fairly broad agreement between theory and experiment in the study ${ }^{5}$ of $\mathrm{Rb}_{2} \mathrm{ZnCl}_{4}$, it is expected that these pair potentials and the intramolecular harmonic expansion will give a reasonable description for the potential energy of the present system.

In order to test the quality of our interionic potentials, we first performed a static energy minimization for the Pna $2_{1}$ structure of $\mathrm{K}_{2} \mathrm{ZnCl}_{4}$. Our relaxation started from the experimental structure ${ }^{10}$ and was subject to the constraints of the Pna $2_{1}$ symmetry. The minimization was for an infinite lattice, obtained by applying periodic boundary conditions, and the standard technique of Ewald summation was used for the calculations of lattice energies and forces, etc. Figure 1 shows the $b c$ cross sections of the experimental (a) and theoretical (b) Pna $2_{1}$ structures. The bonds between a $\mathrm{Zn}$ atom and its four nearest neighbors are shown by straight lines. As can be seen, each of the $\mathrm{ZnCl}_{4}{ }^{2-}$ molecular ions has a $\mathrm{Zn}-\mathrm{Cl}$ bond that is close to being parallel to the $b$ axis. In the high-temperature Pnam structure above the Pna $2_{1}$ phase, these bonds should be exactly parallel to the $b$ axis in projections like Fig. 1 and the three $\mathrm{ZnCl}_{4}{ }^{2-}$ ions that sit along the $a$ axis have identical orientations. Therefore, the tripled Pna $2_{1}$ structure can be seen to be a result of a modulation that mainly rotates the $\mathrm{ZnCl}_{4}{ }^{2-}$ ions about the $a$ axis and correspondingly shifts the $\mathrm{K}^{+}$ions in the $c$ direction. Clearly, the theoretical structure well reproduced this modulation, only the modulation amplitude is 
about $20 \%$ too large. This should not be surprising, since the experimental data are obtained at $T=300 \mathrm{~K}$, while the static relaxed theoretical structure corresponds to $T=0 \mathrm{~K}$. In fact, we have performed a moleculardynamics simulation at $300 \mathrm{~K}$ and found that the modulation amplitude in the average structure is approximately $70 \%$ of that in the static relaxed structure in Fig. 1(b), which is then much closer to that in the experimental structure.

The lattice constants in the relaxed structure are $a=49.143, b=22.205$, and $c=13.447$ a.u., all shorter than the experimental values $(2.9 \%$ for $a, 5.3 \%$ for $b$, and $1.9 \%$ for $c$ ). This has been a rather general feature for large unit-cell simulations using Gordon-Kim potentials and it appeared similarly in our studies ${ }^{4,5}$ of $\mathrm{K}_{2} \mathrm{SeO}_{4}$ and $\mathrm{Rb}_{2} \mathrm{ZnCl}_{4}$. Although these deviations are certainly large in magnitude, they are, however, percentagewise comparable in all three dimensions. The reduced basis parameters in the relaxed structure ${ }^{11}$ are in close agreement with the experimental values. The coordinations of the $\mathrm{Zn}$ and $K$ atoms are reproduced satisfactorily. While larger differences appear between the theoretical and experimental values for some $\mathrm{Cl}$ atoms, they are due to the larger modulation in the theoretical structure as discussed above. Overall, the theoretical structure is more or less uniformly contracted and should still preserve the basic symmetries of the system relevant to the phase transitions under study.

In our previous studies of $\mathrm{K}_{2} \mathrm{SeO}_{4}$ (Ref. 4) and $\mathrm{Rb}_{2} \mathrm{ZnCl}_{4}$, ${ }^{5}$ it was found that the potential-energy surface
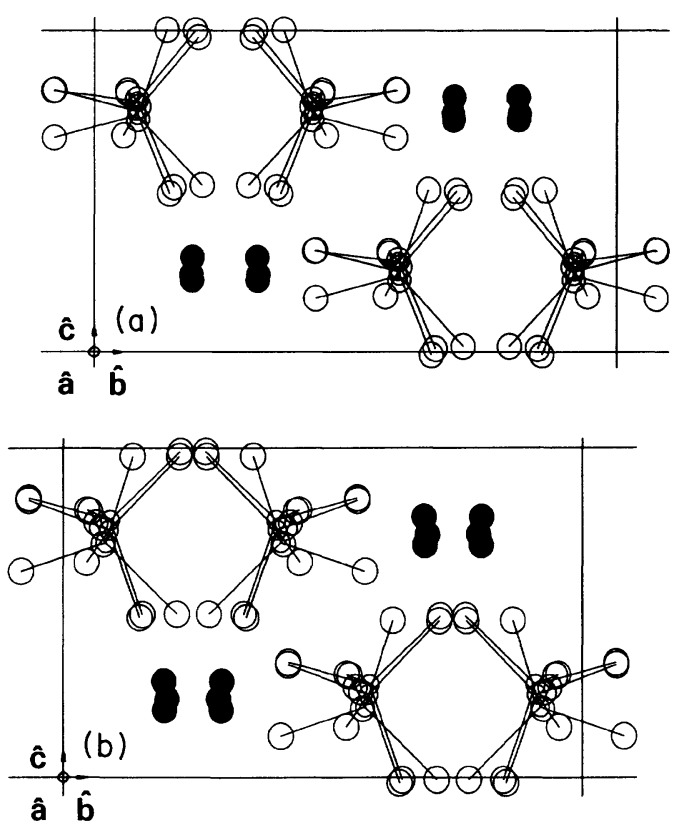

FIG. 1. Projections along the a axis of the (a) experimental and (b) theoretical $P n a 2_{1}$ structures of $\mathrm{K}_{2} \mathrm{ZnCl}_{4}$. Unit vectors indicate the directions of the lattice axes. A unit vector pointing out of the page is represented by a circle. The $\mathrm{Zn}$ and $\mathrm{Cl}$ atoms are represented by small and large open circles, respectively. Bonds (straight lines) connect each $\mathrm{Zn}$ and the four nearest $\mathrm{Cl}$ atoms. The $\mathrm{K}$ atoms are represented by solid circles, except those obscured by the other atoms. pertinent to the phase transitions, i.e., the incommensurate and the subsequent triple lock-in transitions, contains a double well when described using a single Pnam unit cell. On top of the double well is the hightemperature Pnam structure, while at the bottom are two stable monoclinic structures (the angle between the $b$ and $c$ axes $\alpha \neq 90^{\circ}$ ) that have exactly the same potential energy but look like mirror images to each other. These monoclinic structures are the results of a lock-in of the zone-center instability in the Pnam structure. Since $\mathrm{K}_{2} \mathrm{ZnCl}_{4}$ also has the same incommensurate and triple lock-in transitions as $\mathrm{K}_{2} \mathrm{SeO}_{4}$ and $\mathrm{Rb}_{2} \mathrm{ZnCl}_{4}$, we expect to find the same double-well structure on the potential surface. Thus, we performed a static relaxation for the Pnam structure of $\mathrm{K}_{2} \mathrm{ZnCl}_{4}$ and then computed dispersion curves for the relaxed structure. We found, indeed, a zone-center instability just like that in the other two compounds, which leads to exactly the same kind of monoclinic structures as those in $\mathrm{K}_{2} \mathrm{SeO}_{4}$ and $\mathrm{Rb}_{2} \mathrm{ZnCl}_{4}$. Therefore, the potential-energy surface for a single Pnam cell of $\mathrm{K}_{2} \mathrm{ZnCl}_{4}$ indeed has a double-well structure. When multiple cells are involved, this generates a multiple-well structure on the total potential-energy surface which provides the basis for the incommensurate and the cell-tripling phase transitions observed in these systems.

However, the double well in $\mathrm{K}_{2} \mathrm{ZnCl}_{4}$ is deeper than that in $\mathrm{Rb}_{2} \mathrm{ZnCl}_{4}$ and much deeper than that in $\mathrm{K}_{2} \mathrm{SeO}_{4}$. If measured by the difference between the static potential energies of the Pnam structure and of the two energetically equivalent monoclinic structures, the depths for $\mathrm{K}_{2} \mathrm{SeO}_{4}, \mathrm{Rb}_{2} \mathrm{ZnCl}_{4}$, and $\mathrm{K}_{2} \mathrm{ZnCl}_{4}$ are 12.3, 109.8, and $207.3 \mathrm{meV}$ per formula unit, respectively. Moreover, the angles $\alpha$ in the monoclinic structures of $\mathrm{K}_{2} \mathrm{SeO}_{4}$, $\mathrm{Rb}_{2} \mathrm{ZnCl}_{4}$, and $\mathrm{K}_{2} \mathrm{ZnCl}_{4}$ are $88^{\circ}, 84^{\circ}$, and $82^{\circ}$, respectively, indicating that the double well in $\mathrm{K}_{2} \mathrm{ZnCl}_{4}$ is broader than that in $\mathrm{Rb}_{2} \mathrm{ZnCl}_{4}$, and much broader than that in $\mathrm{K}_{2} \mathrm{SeO}_{4}$. Therefore, from $\mathrm{K}_{2} \mathrm{SeO}_{4}$ to $\mathrm{Rb}_{2} \mathrm{ZnCl}_{4}$, and then to $\mathrm{K}_{2} \mathrm{ZnCl}_{4}$, the double well gets deeper and broader. This agrees with the sequence of the Pnam incommensurate transition temperatures in these compounds, ${ }^{1} 129.5$ $\mathrm{K}$ in $\mathrm{K}_{2} \mathrm{SeO}_{4}, 303 \mathrm{~K}$, in $\mathrm{Rb}_{2} \mathrm{ZnCl}_{4}$, and $553 \mathrm{~K}$ in $\mathrm{K}_{2} \mathrm{ZnCl}_{4}$. It is also consistent with the experimental observation ${ }^{12-14}$ that the Pnam phases of both $\mathrm{Rb}_{2} \mathrm{ZnCl}_{4}$ and $\mathrm{K}_{2} \mathrm{ZnCl}_{4}$ are much more disordered than that of $\mathrm{K}_{2} \mathrm{SeO}_{4}$.

The static energy for the relaxed $P n a 2_{1}$ structure is 163 $\mathrm{meV}$ per formula units lower than that for the Pnam structure, but $45 \mathrm{meV}$ higher than that for the singlet monoclinic structures found above. However, in experiment the system transforms from the Pnam phase to the tripled $P$ na $2_{1}$ structure rather than to the singlet structure with a lower static energy. This is very similar to the situations in $\mathrm{K}_{2} \mathrm{SeO}_{4}$ (Ref. 4) and $\mathrm{Rb}_{2} \mathrm{ZnCl}_{4}$, and can be explained with a similar argument. When the temperature is above $550 \mathrm{~K}$ and the system is in the Pnam phase, the relatively small difference in energy between the tripled and the singlet structures makes the two effectively degenerate. Consequently, as the temperature decreases, the Pnam structure begins to transform and 
the state selected will be that of higher entropy. Clearly, this is the Pna $2_{1}$ phase in which the molecular ions have three, rather than one, possible orientations. Subsequently, for the Pna $2_{1}$ structure to transform to the singlet monoclinic structure, it requires that at least one of the tetrahedra switch through the Pnam configuration and, as the system is cooled further, this becomes increasingly difficult. When the temperature is lowered further so that the singlet monoclinic structure becomes preferred energetically, the transformation is frustrated by the $\sim 160-\mathrm{meV}$ barrier and the Pna $2_{1}$ phase persists.

A lattice-dynamics study ${ }^{5}$ of the static relaxed theoretical Pna $2_{1}$ structure of $\mathrm{Rb}_{2} \mathrm{ZnCl}_{4}$ has shown that an instability exists at the point $\mathbf{q}=\left(\mathbf{b}^{*}+\mathbf{c}^{*}\right) / 2$, predicting a soft phonon transition that will double the unit cell along both the $\mathbf{b}$ and $\mathbf{c}$ axes, just as observed in experiment. ${ }^{3}$ We thus calculated the dispersion curves along the [011] direction for the static relaxed theoretical Pna $2_{1}$ structure given [Fig. 1(b)], and plotted them in Fig. 2. The lowest four branches have the same shape as those in $\mathrm{Rb}_{2} \mathrm{ZnCl}_{4}$, but they have positive values at the zone boundary, rather than negative values, indicating that the structure in Fig. 1(b) would not be able to produce a similar lower-temperature structure as in $\mathrm{Rb}_{2} \mathrm{ZnCl}_{4}$, contrary to the experimental fact.

This discrepancy is, however, artificial, if we recall that the theoretical structure in Fig. 1(b) has a much larger cell-tripling modulation amplitude than the experimental structure in Fig. 1(a), due to the fact the former is for $T=0 \mathrm{~K}$, while the latter is for $T=300 \mathrm{~K}$. When we calculate the dispersion curves along the [011] direction for the theoretical static Pnam structure of $\mathrm{K}_{2} \mathrm{ZnCl}_{4}$, we found that the boundary point $q=\left(b^{*}+c^{*}\right) / 2$ is, in fact, unstable, which is found also to be true for $\mathrm{Rb}_{2} \mathrm{ZnCl}_{4}$. Therefore, it is the artificially large modulation from the Pnam structure to the Pna 2 1 structure in Fig. 1(b) that diminishes the $q=\left(b^{*}+c^{*}\right) / 2$ instability in $\mathrm{K}_{2} \mathrm{ZnCl}_{4}$.

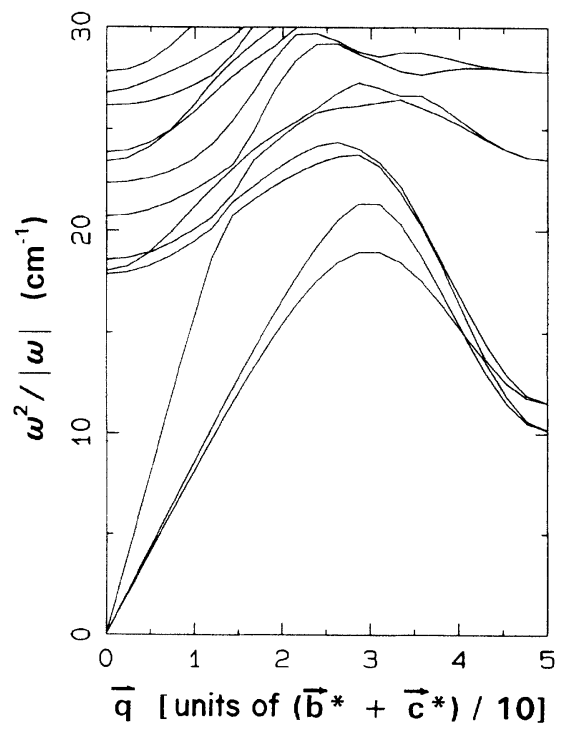

FIG. 2. Dispersion curves along the [011] direction for the theoretical Pna2 $2_{1}$ structure of $\mathrm{K}_{2} \mathrm{ZnCl}_{4}$.
However, in experiment, it is the instability in the structure at $300 \mathrm{~K}$, not at $0 \mathrm{~K}$, that determines the phase diagram.

In order to illustrate this point better, we computed dispersion curves for structures obtained by "stepping" from the theoretical Pnam structure to the theoretical $P n a 2_{1}$ structure linearly. In Fig. 3, we plot the lowest branch of the dispersion curves from the $X$ point $\mathbf{q}=\mathbf{b}^{*} / 2$ to the $T$ point $\mathbf{q}=\left(\mathbf{b}^{*}+\mathbf{c}^{*}\right) / 2$ for each of the Pnam, Pna $2_{1}$, and three intermediate structures I, II, and III representing 25,50 , and $75 \%$ of the total modulation in Fig. 1(b), respectively. We also performed similar calculations for $\mathrm{Rb}_{2} \mathrm{ZnCl}_{4}$ and plot the results in Fig. 4, since experimentally the two compounds behave differently $-\mathrm{K}_{2} \mathrm{ZnCl}_{4}$ has an extra incommensurate phase transition at $144 \mathrm{~K}$ above the cell-doubling transition at $140 \mathrm{~K}^{3}$

In Fig. 3, we see that, even at the intermediate structure III, corresponding to a temperature of about $T=300$ $\mathrm{K}$, the $T$ point is still negative, indicating the instability for the cell-doubling transition. It is, indeed, the last extra $25 \%$ change in the structure that stabilizes the $T$ point. The most interesting result is, however, that in Fig. 3 the minimum on the dispersion curve changes gradually from the $X$ point to the $T$ point, being at 0.42 for structure II and at 0.44 for structure III, while for $\mathrm{Rb}_{2} \mathrm{ZnCl}_{4}$ (Fig. 4) the change is sudden, i.e., no minimum occurred between the $X$ and the $T$ points for any of the dispersion curves. Since structures II and III better represent the actual crystal structure at finite temperatures, this shows that $\mathrm{K}_{2} \mathrm{ZnCl}_{4}$ should have a soft optic phonon-induced incommensurate phase transition with the wave vector $q=0.5 b^{*}+(0.5 \pm \delta) c^{*}$, which is consistent with the experimental observation. ${ }^{3}$

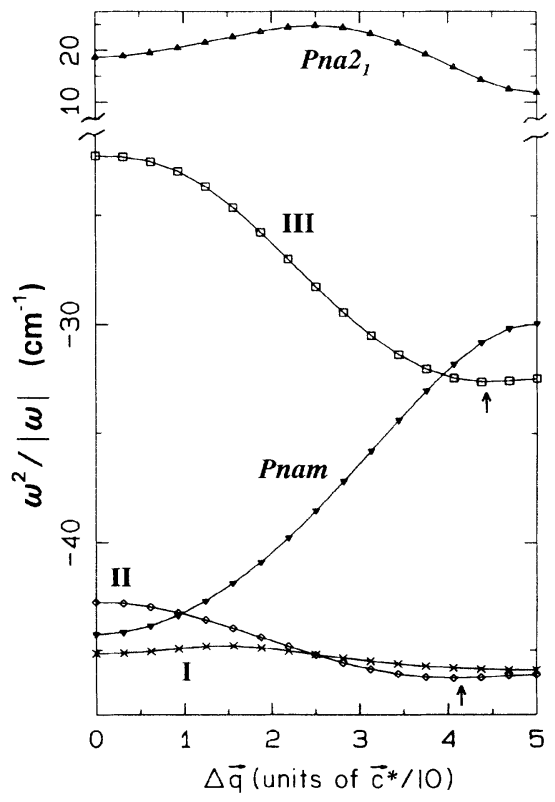

FIG. 3. The lowest dispersion curves from point $\mathbf{b}^{*} / 2$ to point $\left(\mathbf{b}^{*}+\mathbf{c}^{*}\right) / 2$ for the Pnam $(\nabla), \operatorname{Pna} 2_{1}(\triangle)$, and the intermediate structures I $(X)$, II $(\diamond)$, and III $(\square)$ of $\mathrm{K}_{2} \mathrm{ZnCl}_{4}$. The arrows indicate the positions of the minimums on curves II and III. 


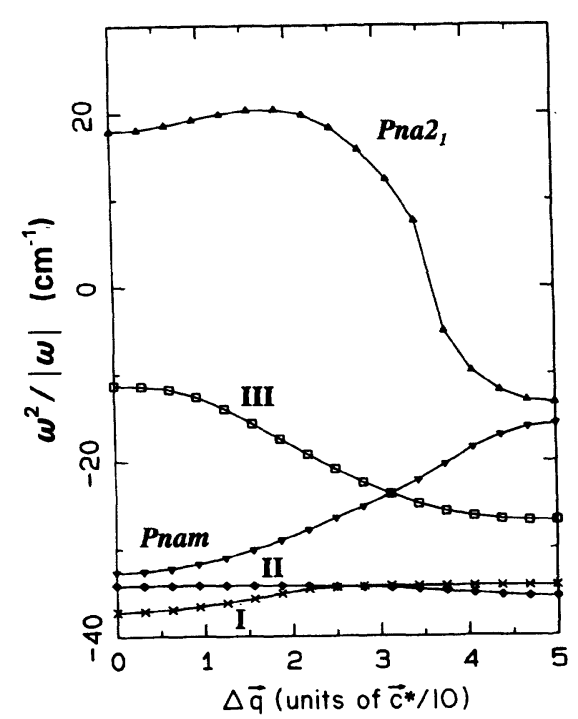

FIG. 4. Same as Fig. 3, except it is for $\mathrm{Rb}_{2} \mathrm{ZnCl}_{4}$.

Hoping to reproduce the lower temperature celldoubling transition, we performed a molecular-dynamics "quench" for a supercell of $\mathbf{6 7 2}$ ions formed by doubling along $b$ and quadrupling along $c$ the $P n a 2_{1}$ unit cell of $\mathrm{K}_{2} \mathrm{ZnCl}_{4}$. Our simulations followed a constant- (zero-) pressure algorithm and did not have any constraints besides the periodic boundary conditions necessary for simulating an infinite crystal. Starting from $T=300 \mathrm{~K}$, the sample was gradually cooled down by reducing the kinetic energy in steps. At each step, averages of atomic positions and lattice angles were taken over a period of 20 psec with the molecular-dynamics step size of $5 \mathrm{fsec}$. It was found that the angle between the a and $\mathbf{c}$ axes started

${ }^{1}$ H. Z. Cummins, Phys. Rep. 185, 211 (1990), and references therein.

${ }^{2}$ K. Gesi, J. Phys. Soc. Jpn. 59, 416 (1990).

${ }^{3}$ M. Quilichini, V. Dvorak, and P. Boutrouille, J. Phys. I France 1, 1321 (1991).

${ }^{4}$ H. M. Lu and J. R. Hardy, Phys. Rev. Lett. 64, 661 (1990); Phys. Rev. B 42, 8339 (1990).

${ }^{5}$ H. M. Lu and J. R. Hardy, Phys. Rev. B 45, 7609 (1992).

${ }^{6}$ R. G. Gordon and Y. S. Kim, J. Chem. Phys. 56, 3122 (1972).

${ }^{7}$ M. Quilichini and J. Pannatier, Acta Crystallogr. B 39, 657 (1983).

${ }^{8}$ M. Quilichini, G. Heger, and P. Schweiss, Ferroelectrics 79, to deviate from $90^{\circ}$ around $T=250 \mathrm{~K}$, and increased to $90.7^{\circ}$ at $20 \mathrm{~K}$, while the other two lattice angles remained at $90^{\circ}$ at all temperatures. Therefore, we have produced a monoclinic phase transition. But the average structure at the lower temperature was neither doubled in b nor in $\mathbf{c}$, contrary to the experimental observation. ${ }^{3}$

We tend to believe that this discrepancy should be attributed to the limitations of the molecular-dynamics calculations, rather than to the quality of the $a b$ initio interionic potentials; these potentials should be as good as those used in treating ${ }^{5} \mathrm{Rb}_{2} \mathrm{ZnCl}_{4}$ where close agreement with experiment was obtained for all the phase transitions, since the same description of the electron charge density of the $\mathrm{ZnCl}_{4}{ }^{2-}$ ion is used. One possible explanation could be that there are many metastable configurations that are very close in energy to the true ground state so that the molecular dynamics for a limited phase space and limited equilibration time is simply inadequate. In fact, this was the case for $\mathrm{Rb}_{2} \mathrm{ZnCl}_{4}$, where the similar molecular-dynamics "quench" resulted in a "wrong" ground-state structure with the monoclinic angle too large, although the structure was indeed doubled along both $b$ and $c$ directions. ${ }^{5}$ Naturally, there may be other equally probable explanations as well. But, in any case, to clarify the situation requires simulations involving even longer equilibrium times and a still larger supercell, and is thus beyond the scope of the present report. Alternately, it may be that, since our supercell does not permit the new phase incommensurate along $c$ to form, it also rules out formation of the commensurate $T$ point modulation if the former is an essential precondition for the latter to occur.

This work was supported by the U.S. Army Research Office.
117 (1988).

${ }^{9}$ E. Clementi and C. Roetti, At. Data Nucl. Data Tables 14, 177 (1974).

${ }^{10}$ I. Mikhail and K. Peters, Acta Crystallogr. B 35, 1200 (1979).

${ }^{11}$ Available on request.

${ }^{12}$ N. E. Massa, F. G. Ullman, and J. R. Hardy, Phys. Rev. B 27, 1523 (1983).

${ }^{13}$ K. Itoh, A. Hinisada, H. Matsunaga, and E. Nakamura, J. Phys. Soc. Jpn. 52, 664 (1983).

${ }^{14}$ V. Katkanant, J. R. Hardy, R. D. Kirby, and F. G. Ullman, Ferroelectrics 99, 213 (1989). 